Research Journal of Applied Sciences 5 (6): 388-393, 2010

ISSN: $1815-932 \mathrm{X}$

(C) Medwell Journals, 2010

\title{
Incorporating Decision Making Capability into the Social Force Model in Unidirectional Flow
}

\author{
Zarita Zainuddin and Mohammed Shuaib \\ School of Mathematical Sciences, University Sains Malaysia, 11800 USM, Pulau Penang, Malaysia
}

\begin{abstract}
The supremacy of microscopic models in pedestrian studies has been evaluated on their ability to reproduce the self-organization phenomenon of pedestrian flow. The social force model which is characterized by considering the pedestrians as self-driven particles has preceded many others in presenting these phenomena. Over the last few years, aspects of decision making capability have been incorporated gradually into the model in both normal and evacuation situations. However, the decision making aspects in social force model is still in infant stage. In this study, the social force model is extended by incorporating the investigation ability for the independent pedestrians as an aspect of decision making capability. Afterwards, by performing simulations, we examine qualitatively the effect of activating the investigation ability for the independent pedestrians on their motion in a unidirectional walkway compared with the original model.
\end{abstract}

Key words: Social force model, investigation ability, pedestrian flow, capability, supremacy, phenomenon

\section{INTRODUCTION}

Over the last few decades, researchers have devoted much attention to pedestrian studies to provide solutions for some pressing problems such as congestion (Teknomo, 2002). Microscopic studies which are essentially a branch of pedestrian studies are mainly concerned with the interactions among pedestrians and their effects upon each other (Teknomo, 2002). One of the most important models in the microscopic level of pedestrian studies is the social force model. The most important feature in this model is the representation of a pedestrian's motivations exerted by other objects (pedestrians and obstacles) surrounding him as social forces. The sum of these forces is implemented in a Newtonian equation. The main equations of the model are:

$$
\begin{gathered}
\frac{d \overrightarrow{\mathrm{x}}_{\mathrm{i}}(\mathrm{t})}{\mathrm{dt}}=\vec{v}_{\mathrm{i}}(\mathrm{t}) \\
\mathrm{m}_{\mathrm{i}} \frac{\mathrm{d} \vec{v}_{\mathrm{i}}}{\mathrm{dt}}=\overrightarrow{\mathrm{f}}_{\mathrm{i}}+\varepsilon_{\mathrm{i}}=\overrightarrow{\mathrm{f}}_{\mathrm{i}}^{\text {pref }}+\sum_{\mathrm{j}} \overrightarrow{\mathrm{f}}_{\mathrm{ij}}+\sum_{\text {object }} \overrightarrow{\mathrm{f}}_{\mathrm{i} \text { object }}+\varepsilon_{\mathrm{i}} \\
\overrightarrow{\mathrm{f}}_{\mathrm{i}}^{\text {pref }}(\mathrm{t}):=\frac{\mathrm{m}_{\mathrm{i}}}{\tau}\left(\vec{v}_{\mathrm{i}}^{0}(\mathrm{t})-\vec{v}_{\mathrm{i}}(\mathrm{t})\right) \\
\overrightarrow{\mathrm{v}}_{\mathrm{i}}^{0}=\overrightarrow{\mathrm{e}}_{\mathrm{i}}^{0}\left(1+\mathrm{E}_{\mathrm{i}}\right) \mathrm{V}_{\mathrm{i}}^{0} \mathrm{D}_{\mathrm{i}}+\left\langle\vec{v}_{\mathrm{j}}\right\rangle_{\mathrm{i}}\left(1-\mathrm{D}_{\mathrm{i}}\right) \\
\overrightarrow{\mathrm{f}}_{\mathrm{ij}}(\mathrm{t})=\overrightarrow{\mathrm{f}}_{\mathrm{ij}}^{\text {att }}(\mathrm{t})+\overrightarrow{\mathrm{f}}_{\mathrm{ij}}^{\text {rep }}(\mathrm{t})+\overrightarrow{\mathrm{f}}_{\mathrm{ij}}^{\text {push }}(\mathrm{t})+\overrightarrow{\mathrm{f}}_{\mathrm{ij}}^{\text {friction }}(\mathrm{t})
\end{gathered}
$$

$$
\overrightarrow{\mathrm{f}}_{\mathrm{i}, \mathrm{object}}(\mathrm{t})=\overrightarrow{\mathrm{f}}_{\mathrm{i}, \mathrm{abject}}^{\text {att }}(\mathrm{t})+\overrightarrow{\mathrm{f}}_{\mathrm{i}, \mathrm{object}}^{\mathrm{rep}}(\mathrm{t})+\overrightarrow{\mathrm{f}}_{\mathrm{i}, \mathrm{bbject}}^{\text {push }}(\mathrm{t})+\overrightarrow{\mathrm{f}}_{\mathrm{i}, \mathrm{bbject}}^{\text {ffiction }}(\mathrm{t})
$$

Where:

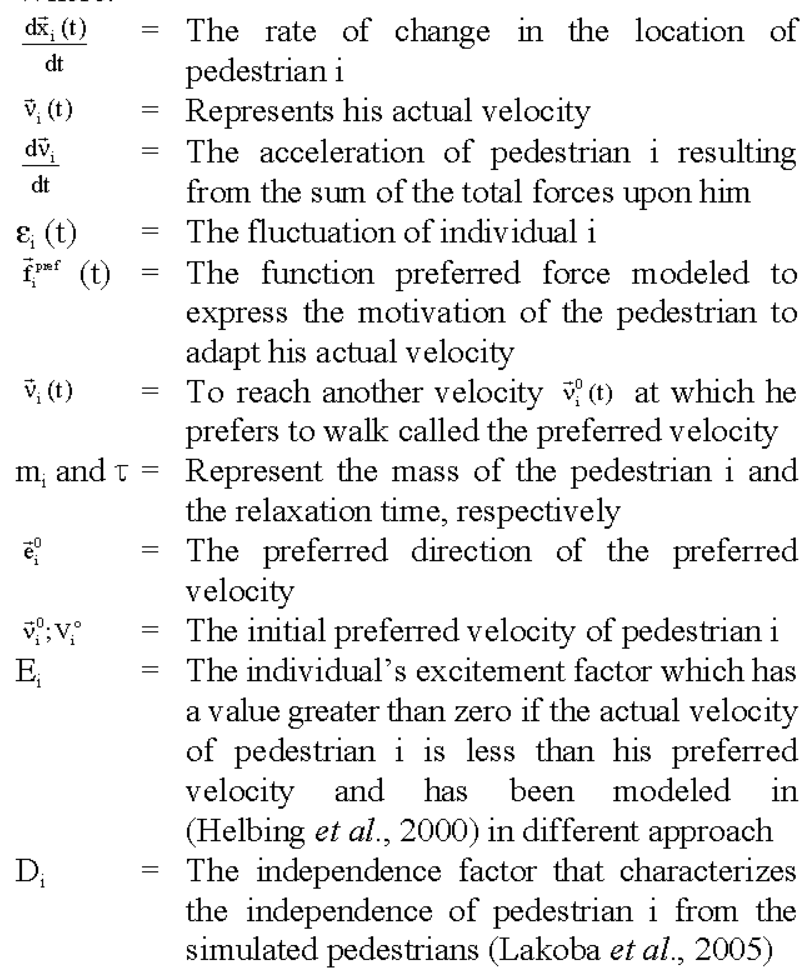

In addition, the function $\overrightarrow{\mathrm{f}}_{\mathrm{ij}}(\mathrm{t})$ is the sum of all forces exerted by pedestrian $\mathrm{j}$ upon pedestrian $\mathrm{i}$ which are

Corresponding Author: Zarita Zainuddin, School of Mathematical Sciences, University Sains Malaysia, 11800 USM, Pulau Penang, Malaysia 
of two types. The first type are the social forces, the repulsion force $\overrightarrow{\mathrm{f}_{i j}^{\text {mp }}}(t)$ and the attraction force $\overrightarrow{\mathrm{f}}_{\mathrm{ij}}^{\text {att }}(\mathrm{t})$ that represent the model of the repulsive and attractive motivations inside pedestrian $\mathrm{i}$ against and towards $\mathrm{j}$, respectively (Helbing and Molnar, 1995). The second type are the physical forces $\overrightarrow{\mathrm{f}}_{\mathrm{y}}^{\text {pesh }}(\mathrm{t})$ and $\overrightarrow{\mathrm{f}}_{\mathrm{fi}}^{\text {firtion }}$ (t) which were modeled as linear functions in (Helbing et al., 2000) in analogy with the granular forces; $\overrightarrow{\mathrm{f}}_{\mathrm{i}, 0 j \mathrm{ject}}(\mathrm{t})$ is analogous to $\overrightarrow{\mathrm{f}}_{\mathrm{ij}}(t)$ but with regards to walls.

\section{DECISION MAKING ASPECTS IN SOCIAL FORCE MODEL}

Incorporating decision-making aspects into the model has been initialized (Lakoba et al., 2005) by granting the pedestrians the independence factor $\mathrm{D}$ which helps in granting the pedestrians with some kind of intelligence when making decisions. For evacuation situations, the researchers (Zainuddin and Shuaib, 2009, 2010) have pursued the last research by incorporating more aspects of intelligence such as the familiarity factor to let the independent pedestrians recognize the exits that actually lead to safety from the available alternative exits by granting him the ability in performing an assessment process. Moreover, they incorporated the leader factor (Zainuddin and Shuaib, 2010) as an aspect for dependent pedestrians: adhering to others and following their leaders locations. Examples of those are the children and the elders who totally depend on their close relatives such as parents with whom they are walking. However, while performing simulations for the motion in normal situation, a counterintuitive behavior has emerged that the simulated pedestrians are too short-sighted to avoid some anticipated blocked situations especially because of unevenly crowded areas (Fig. 1). The pedestrians have no ability to avoid such anticipated blocked situations before joining these situations. Indeed, the pedestrian in social force model has limited perception imposed by the repulsive distance parameter $\beta^{\text {rep }}$ used in the repulsive social force modeled (Helbing and Molnar, 1995) by:

$$
\vec{f}_{i j}^{\text {rep }}(t):=A^{\mathrm{rep}} \mathrm{e}^{\left(R_{\mathrm{ij}}-d_{\mathrm{ij}}(\mathrm{t})\right) / \mathrm{B}^{\mathrm{eep}}} \overrightarrow{\mathrm{n}}_{\mathrm{ij}}
$$

Where:

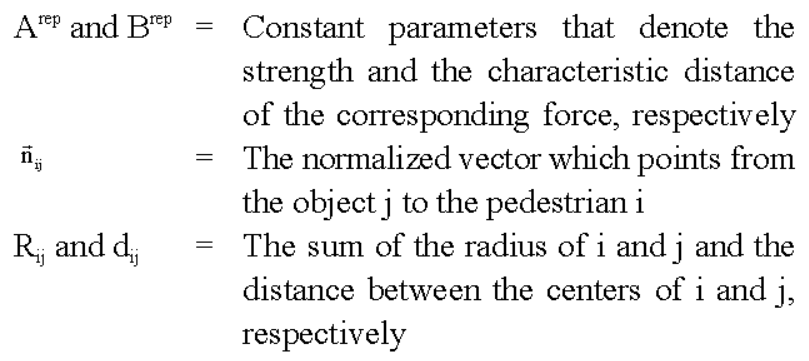

As shown in Fig. 2, the perception area of a pedestrian is the circular area centered at his location where any entity inside exerts a social force which affect his motion. Any entity beyond this area has a very small magnitude of force that would not affect the relevant pedestrian's motion. The last renders the pedestrian a short-sighted walker. In reality, as these anticipated blocked situations lies within his sight, the pedestrian has further ability to make a decision to avoid such situations. The model of the investigation capability is based on

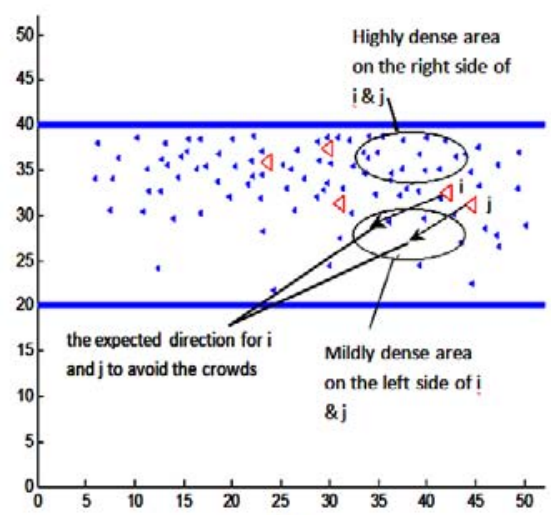

a)

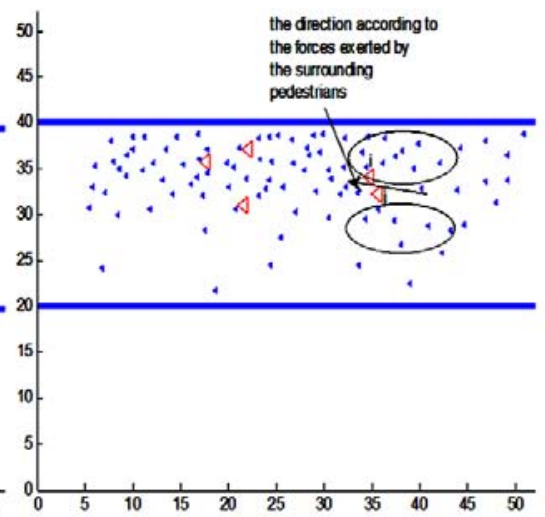

b)

Fig. 1: The simulation of a unidirectional walkway for pedestrians walking from the right side to their destination which is on the left side. The big triangles represent independent pedestrians with a higher desired velocity than those who are represented by the small triangles. The arrows in (a) represent the expected direction for $i$ and $j$ because the natural behavior is to avoid the crowds by walking in less dense areas especially if they are uncomfortable (walking less than their desired velocity). In (b) they do not have the ability to make a decision, so their behaviour governed by the forces exerted by the surrounding pedestrians 


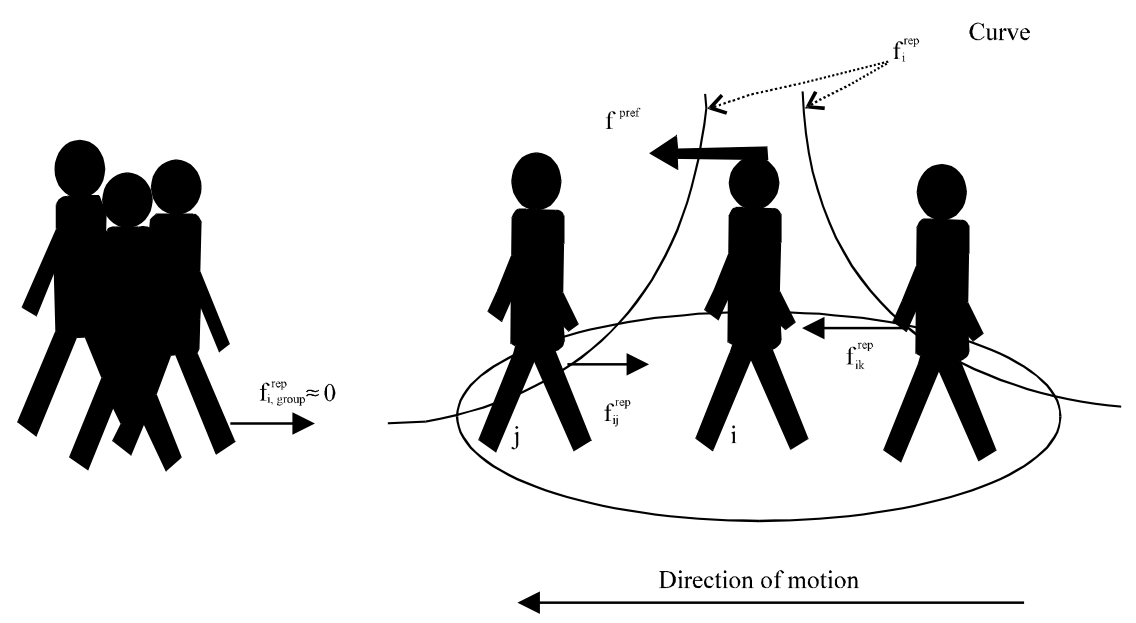

Fig. 2: The perception of a pedestrian is represented as a circle centered at the location of the pedestrian himself. It is an estimated area that the repulsive forces exerted by the other pedestrians have significant values. The reason of this is the exponential function of the repulsive social force as modeled by Helbing and Molnar (1995)

the fact that the independent pedestrian has the ability to investigate the situation outside the limited perception given by the social force model.

\section{THE INVESTIGATION CAPABILITY MODEL}

With the investigation capability, the pedestrian can make a decision to avoid some expected difficult situations. In other words, the pedestrian directs his motion toward a less dense area as an intermediate area to his destination (Fig. 3). The main components of the investigation capability are the investigation area (the area of his sight), the investigation process and the decision making.

Modelling the investigation area: In reality, the independent pedestrian has an ability to investigate the macroscopic behaviors of other pedestrians who are located within his sight (the investigation area) such as the pedestrian density. The investigation area can be modeled in many forms to represent the pedestrian's sight. Here, it is preliminary represented by a rectangle shape and divided into three parts: the left part, the forward part and the right part as shown in Fig. 4. They are indexed by part 1-3, respectively. It is also assumed that the walkway is wide enough and the destination is far away enough to neglect the geometrical computational dynamics of the investigation area. Many examples of such physical environments are present in the Al-hajj area in Makkah (Fig. 3) and has received significant attention from researchers (Helbing et al., 2007). The pedestrian will choose one of these parts to determine his next route. Accordingly, the three potential routes which are introduced as representatives for the relevant parts are assumed to constitute the pedestrian's alternative routes as shown in Fig. 4. Basically, the forward route (route 2) at time $t$ is defined by the straight link that connects the pedestrian directly to his destination, according to the directness principle (Helbing, 1997). Accordingly, the investigation area is dynamic corresponding to the dynamic changes in the pedestrian's location.

Modeling the investigation process and the decision: The model for the investigation process is developed by taking into account the repulsive characteristics of each part (such as the average density inside) and its effect on the repulsion of the relevant routes. The decision making model is developed by adopting the minimally repulsive route. The ability to start the investigation process is granted to the independent pedestrian by the factor $\operatorname{Inv}_{\mathrm{i}}$ :

$$
\operatorname{Inv}_{\mathrm{i}}=1 \text { if } \mathrm{E}_{\mathrm{i}} \geq 0
$$

where, $E_{i}$ is the excitement factor. The model for the repulsion of the alternative routes for pedestrian $i$ who becomes engaged with this process (i.e., Inv $=1$ ) is:

$$
\operatorname{rep}_{\text {routei }, j}(t)=\sigma_{p a r t}(t)
$$

where, rep $_{\text {sout }_{i, j}}$ is the repulsive effect of route $\mathrm{j}$ included in part $\mathrm{j}$. The index of the route with the minimum repulsion is determined by comparison between the computed repulsions of the alternative routes and selecting the minimum repulsive route: 
Res. J. Applied Sci., 5 (6): 388-393, 2010

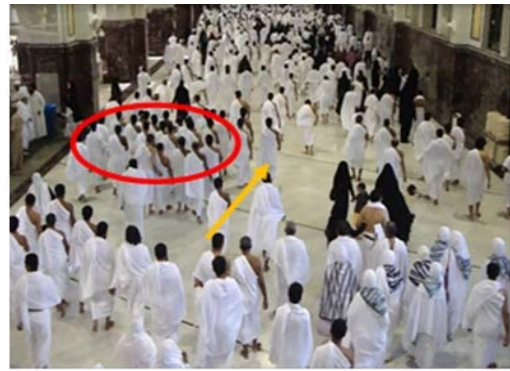

(a)

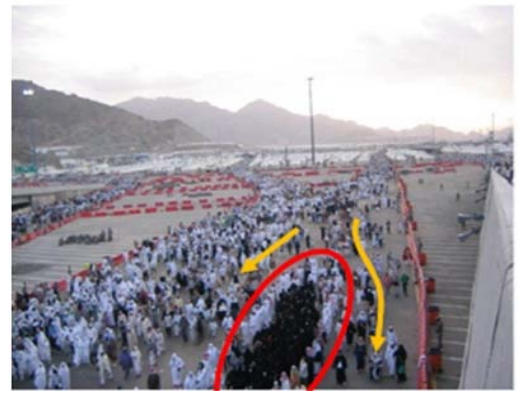

(c)

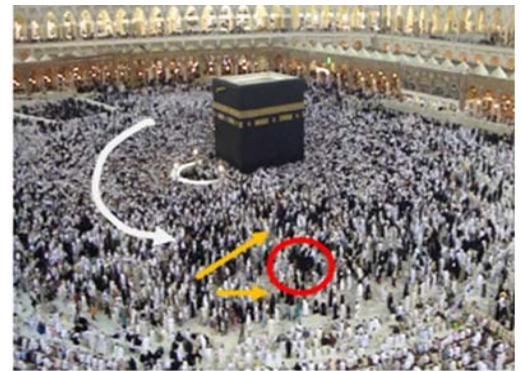

(b)

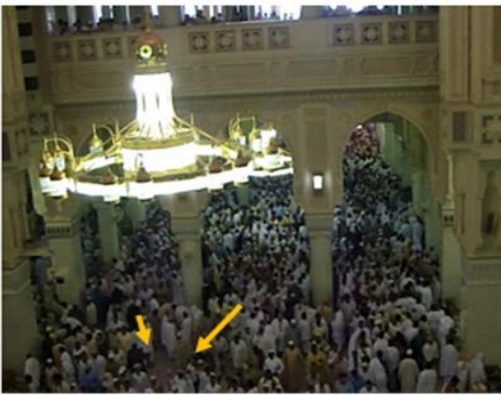

(d)

Fig. 3: Aspects of Muslims rituals done in the Al-hajj area in Makkah where the dominant situation is a unidirectional pedestrian flow

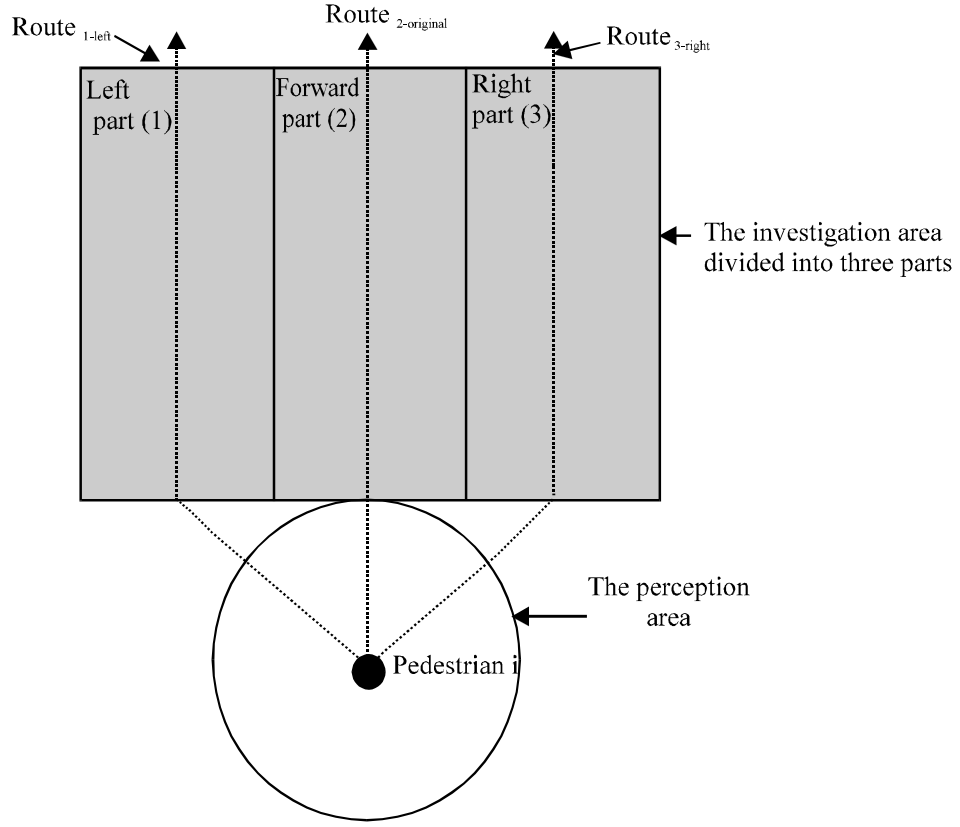

Fig. 4: The perception area represented by a circle and the investigation area represented by three rectangular parts, each part includes a representative route

$$
\text { new, } i=\text { index }\left\{\min _{\mathrm{j}}\left(\operatorname{rep}_{\text {route }_{i, j}}(\mathrm{t})\right) \mid \mathrm{j} \in\{1,2,3\}\right\}
$$

Finally, the pedestrian makes a decision to direct his motion toward a new area which contains the route that has the minimum repulsion rep new, $_{i}$ if the new route provides a utility to the pedestrian with worthwhile magnitude $g$ where:

$$
D C_{i}=\left\{\begin{array}{cc}
1 & \text { rep }_{\text {curr, },}-\text { rep }_{\text {new, },} \geq g \text { and } \operatorname{Inv}_{\mathrm{i}}=1, \\
0 & \text { otherwise }
\end{array}\right.
$$


Where $\mathrm{g}$ differs from one pedestrian to another according to psychological aspects. The desired direction of the pedestrian in Eq. 4 is replaced by $\vec{e}_{\mathrm{cur}, \mathrm{i}}^{0}$ and Eq. 4 becomes:

$$
\begin{array}{r}
\vec{v}_{\mathrm{i}}^{0}=\overrightarrow{\mathrm{e}}_{\text {curr }, \mathrm{i}}^{0}\left(1+\mathrm{E}_{\mathrm{i}}\right) \mathrm{V}_{\mathrm{i}}^{0} \mathrm{D}_{\mathrm{i}}+\left\langle\vec{v}_{\mathrm{j}}\right\rangle_{\mathrm{i}}\left(1-\mathrm{D}_{\mathrm{i}}\right) \\
\overrightarrow{\mathrm{e}}_{\text {cur }, \mathrm{i}}^{0}(\mathrm{t})= \begin{cases}\overrightarrow{\mathrm{e}}_{\mathrm{new}, \mathrm{i}}^{0} & \mathrm{DC}_{\mathrm{i}}=1 \\
\overrightarrow{\mathrm{e}}_{\mathrm{cur}, \mathrm{i}}^{0} & \mathrm{DC} C_{\mathrm{i}}=0\end{cases}
\end{array}
$$

The pedestrian continues investigating the situation except in the case when he is directly walking toward his destination when the excitement factor is equals to zero.

\section{SIMULATION}

The purpose of this section is to perform a simulation to trace the behavior of pedestrians who are independent and have the investigation feature. The physical environment shown in Fig. 5 is set up according to the assumptions that have been given above: a wide unidirectional walkway that does not include attractive or repulsive sources (such attractive and repulsive sources are planned to be incorporated in future refinement of the model).

The area of origin of the pedestrians is on the right side of the walkway and the destination is on the other side and is far away enough that the preferred direction seems to be horizontal.

The situation is normal and the pedestrians differ in their velocities. Five pedestrians have higher desired velocities than the velocities of the surrounding pedestrians are represented in the diagram by larger triangles than the others and are generated in the upper part of the generator area.

The criterion to validate the above research is the appearance of lane-changing behavior that is the last pedestrians who are walking in inconvenient lanes will
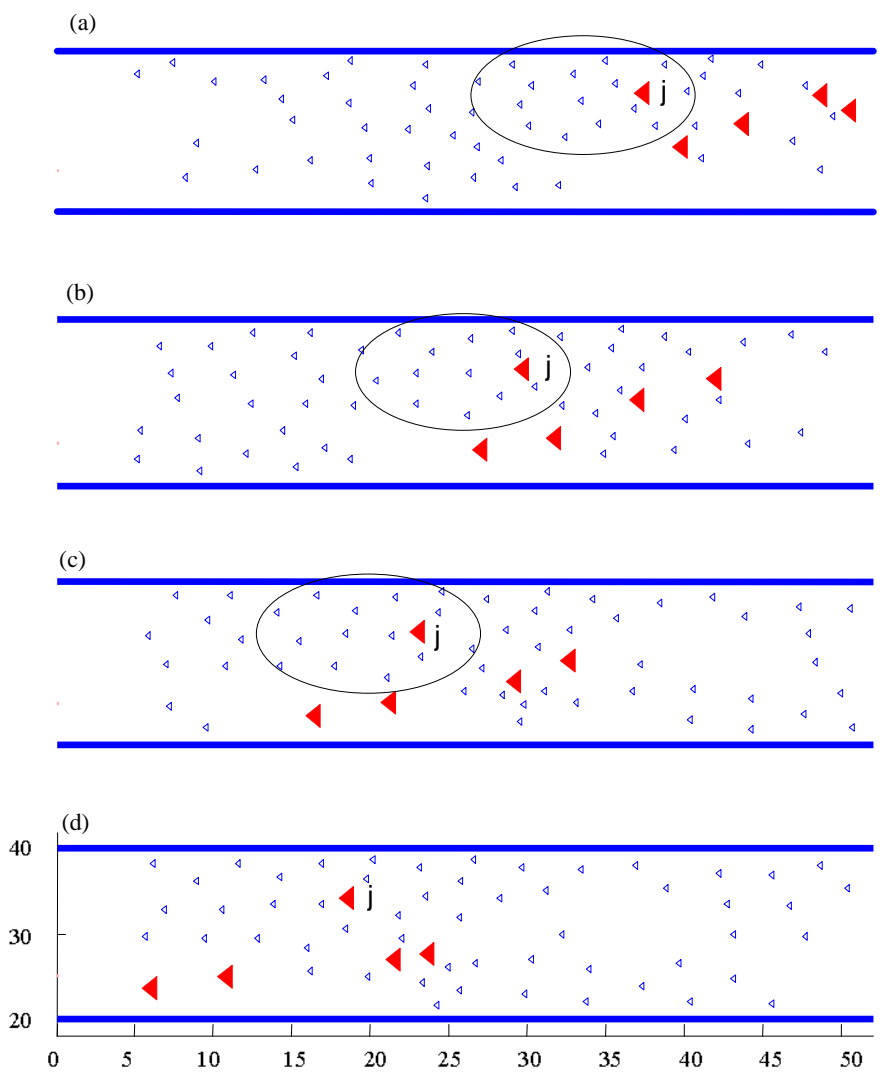

Fig. 5: Four consecutive snapshots of the walkway. The pedestrians who have normal velocity are represented by small triangles and the high-velocity independent pedestrians are represented by larger triangles. When tracing the pedestrians with high velocities (the upper part of the walkway), they were changing their lane as the result of their investigation process of the forward area in which they were looking for a less dense area. Pedestrian $\mathrm{j}$ did not change his lane because the utilities of the areas under his investigation did not make it worthwhile 
Res. J. Applied Sci., 5 (6): 388-393, 2010

Table 1: The pedestrians parameters, the social force model parameters and the extended model parameters

\begin{tabular}{|c|c|}
\hline Camparison & Description \\
\hline \multicolumn{2}{|c|}{ The pedestrians parameters } \\
\hline $\mathrm{m}=[77-83] \mathrm{kg}$ & The pedestrians mass: Uniformly distributed within the range $77-83 \mathrm{~kg}$ \\
\hline $\mathrm{r}=[0.25-0.30] \mathrm{m}$ & The pedestrians radius: uniformly distributed within the range $0.25-0.30 \mathrm{~m}$ \\
\hline \multicolumn{2}{|c|}{ The parameters of the social force model } \\
\hline $\mathrm{A}^{\mathrm{rep}}=2000 \mathrm{~N}$ & The strength of the repulsive social force \\
\hline $\mathrm{B}^{\mathrm{rep}}=0.15$ & The characteristic repulsive distance of the repulsive force \\
\hline $\mathrm{k}=1.4 \times 105 \mathrm{~kg} \mathrm{sec}^{-2}$ & The strength of the contact (pushing) force \\
\hline$\kappa=1$ & The coefficient of the friction force \\
\hline$\tau=0.5 \mathrm{sec}$ & The pedestrian reaction time \\
\hline$\varepsilon \in\left[v^{0} / \tau, 0.05^{*} v^{0} / \tau\right]$ & $\begin{array}{l}\text { The fluctuation source of the pedestrian's acceleration is randomly } \\
\text { assigned to each individual }\end{array}$ \\
\hline \multicolumn{2}{|c|}{ The extended model parameters } \\
\hline Uth $=0.2^{*} \mathrm{rep}_{\text {cur }}$ & The threshold of utility to make a decision \\
\hline Eth $=0.2$ & The threshold of excitement \\
\hline Sth $=1$ & The threshold of density pressure \\
\hline$z=0.3$ & Constant parameter \\
\hline $\mathrm{h}\left(\mathrm{so}_{\overline{\mathrm{c}}}, \mathrm{soc}_{\mathrm{j}}\right)=0$ & The social attribute function \\
\hline
\end{tabular}

change lanes to more appropriate ones. In view of this criterion, the pedestrians we are tracing are those who have a high velocity compared with surrounding pedestrians and who change their direction toward a lower density area to conserve their velocity and walk comfortably. As we are tracing a qualitative aspect, the value of the parameters plays no important role in determining this aspect. However, the parameters used in our model are shown in Table 1. Because of the shortage of psychological studies, $x$ th, Uth and $z$ are chosen to be identical for all pedestrians and their values are estimated by performing the simulation many times and obtaining the best behavior (qualitative behavior) based on the observations in Fig. 3 and the intuition.

\section{CONCLUSION}

In this study, a new aspect of decision-making has been incorporated into the social force model of pedestrian behavior. The pedestrians explore the area under their sights to avoid some predicted situations such as crowded areas or to look for more comfortable lanes to walk in. However, the research of this study can be easily extended to involve many aspects of pedestrian flow by considering multi-directional flow and incorporating some aspects such as a semi-blocked situation or physical characteristics such as obstacles and walls. Density and the height of pedestrians have major roles in modeling the dimension of the investigational lattice and it is recommended to incorporate these factors by conducting relevant empirical studies.

\section{ACKNOWLEDGEMENT}

Researchers thank the University Sains Malaysia for supporting this research under the USM Fellowship, USM-RU-PRGS and ARU Grants.

\section{REFERENCES}

Helbing, D. and P. Molnar, 1995. Social force model for pedestrian dynamics. Phys. Rev. E. Stat. Phys. Plasmas Fluids Relat. Interdiscip. Topics, 51: 4282-4286.

Helbing, D., 1997. Traffic Dynamics: New Physical Modelling Concepts. Springer-Verlag, Berlin, Gemany, (In German).

Helbing, D., A. Johansson and H.Z. Al-Abideen, 2007. Dynamics of crowd disasters: An empirical study. Phys. Rev., E 75: 046109-046109.

Helbing, D., I. Farkas and T. Vicsek, 2000. Simulating dynamical features of escape panic. Nature, 407: 487-490.

Lakoba, T.I., D.J. Kaup and N.M. Finkelstein, 2005. Modifications of the helbing-molnar-farkas-vicsek social force model for pedestrian evolution. Simulation, 81: 339-352.

Teknomo, K., 2002. Microscopic pedestrian flow characteristics: Development of an image processing data. Ph.D. Thesis, Tokyo University, Japan.

Zainuddin, Z. and M. Shuaib, 2009. Modeling the independence factor and its effect on the preferred force of the social force model in emergency and non-emergency situations. Applied Math. Inform. Sci., (In Press).

Zainuddin, Z. and M. Shuaib, 2010. Modification of the decision making capability in the social force model for the evacuation process. Transport Theory Statist. Physics (In Press) 